\title{
Indução do florescimento de Hemerocallis hybrida cv. Graziela Barroso pela aplicação de ácido giberélico ${ }^{1}$
}

\section{Flowering induction of Hemerocallis hybrida cv. Graziela Barroso by gibberellic acid application}

\author{
Michelle Melissa Althaus-Ottmann ${ }^{2 *}$; Ricardo Serra Borsatto ${ }^{3}$; Katia Christina \\ Zuffellato-Ribas ${ }^{4}$; Henrique Soares Koehler ${ }^{5}$; Nilce Nazareno da Fonte ${ }^{6}$
}

\begin{abstract}
Resumo
A produção de Hemerocallis spp., planta muito utilizada no paisagismo, que floresce naturalmente na primavera, tem destaque em Santa Catarina. O objetivo do presente trabalho foi estudar a indução do florescimento em Hemerocallis hybrida cultivar Graziela Barroso, pela aplicação ácido giberélico (0 $\mathrm{mgL}^{-1}$ controle; $15 \mathrm{mgL}^{-1} ; 30 \mathrm{mgL}^{-1} \mathrm{de} \mathrm{GA}_{3}$ ), no verão (janeiro-abril), outono (abril-julho) e inverno (julho-outubro), de forma a aprimorar seus atributos comerciais. Foi utilizado um delineamento experimental inteiramente casualizado, com fatorial 3x10 (três doses de $\mathrm{GA}_{3}$ e dez datas de aplicação para cada estação). Houve interação entre os fatores estudados apenas para acúmulo de massa seca (verão), sendo o melhor valor encontrado $\left.13,81{\mathrm{~g}\left(30 \mathrm{mgL}^{-1} \text { de GA }\right.}_{3}\right)$, na aplicação de 16 de abril; e área foliar (outono), $436,3 \mathrm{~cm}^{2}$ (30 $\mathrm{mg} \mathrm{L}^{-1} \mathrm{de} \mathrm{GA}_{3}$ ) na aplicação de 1 de julho. Não se pode indicar o $\mathrm{GA}_{3}$ nas concentrações estudadas para aprimorar atributos comerciais (florescimento e crescimento) na espécie em questão.
\end{abstract}

Palavras-chave: hemerocale, giberelina, plantas ornamentais

\begin{abstract}
Hemerocallis spp. production is important in Santa Catarina State. The flowers have a great use in landscaping, and bloom in spring. This work had the aim of studying the flower induction in Hemerocallis hybrida cv Graziela Barroso by different applications of gibberellic acid $\left(0 \mathrm{mgL}^{-1}\right.$ control; $15 \mathrm{mgL}^{-1} ; 30$ $\mathrm{mgL}^{-1} \mathrm{GA}_{3}$ ) during summer, autumn and winter. A completely randomized design was used. Treatments represented a factorial arrangement of three $\mathrm{GA}_{3}$ concentrations and ten application occasions in each season. Interaction between the factors was evaluated by the following variables: dry matter (summer) the best value found was 13,81 $\mathrm{g}$ (in $30 \mathrm{mgL}^{-1} \mathrm{GA}_{3}$ ) in April, 16th application, and foliar area (autumn) $436,3 \mathrm{~cm}^{2}$ (in $30 \mathrm{mgL}^{-1} \mathrm{GA}_{3}$ ) in July, $1^{\text {st }}$ application. The gibberellic acid cannot be indicated to increase the commercial qualities (blooming and growing) of Hemerocallis hybrida cv Graziela Barroso.
\end{abstract}

Key words: daylily, gibberellin, ornamental plants

${ }^{1}$ Artigo referente a Dissertação de Mestrado, apresentado à Disciplina de Complexidade e Interdisciplinaridade, do curso de PósGraduação Produção Vegetal, UFPR.

2 Doutoranda em Agronomia - Produção Vegetal, Depto. Fitotecnia e Fitossanitarismo, Universidade Federal do Paraná, UFPR. E-mail: michellealthaus@hotmail.com

3 Doutorando em Engenharia Agrícola, UNICAMP (SP). E-mail: rsborsat@ig.com.br.

${ }^{4}$ Profa. Dra., Depto. Botânica, Universidade Federal do Paraná, UFPR, Curitiba (PR). E-mail: kazu@ufpr.br.

${ }_{5}^{5}$ Prof. Dr., Depto. Fitotecnia e Fitossanitarismo, Universidade Federal do Paraná, UFPR, Curitiba (PR). E-mail: koehler@ufpr. br.

6 Profa. Dra., Depto. Farmácia, Universidade Federal do Paraná, UFPR, Curitiba (PR). E-mail: nilce@ufpr.br

* Autor para correspondência 


\section{Introdução}

A região sul do Brasil representa uma parcela significativa na produção e comercialização nacional de flores e plantas ornamentais, destacando-se o estado de Santa Catarina, onde a produção de plantas para paisagismo tem uma longa tradição, que se iniciou a partir dos imigrantes europeus (CASTAN, 2002).

Esta produção cresceu significativamente, com a criação da APROESC (Associação de Produtores de Plantas Ornamentais de Santa Catarina), associação com mais de 500 associados, que promove o desenvolvimento da cadeia produtiva, desde o produtor até o varejista e o jardineiro. Outro ponto favorável também deve-se ao aparecimento de produtores com forte especialização em poucos produtos, como no caso dos Hemerocallis (CASTAN, 2002).

Hemerocallis spp. são plantas herbáceas, rizomatosas, perenes, acaules, nativas da Europa e Ásia, com 40-60 cm de altura, muito utilizadas no paisagismo, principalmente para revestimento de bordaduras, são cultivadas a pleno sol e florescem significativamente melhor em dias longos (primavera-verão) do que em dias curtos (outonoinverno), o que as classifica como "plantas de dia longo" (PDL) (HERTOGH; LE NARD, 1993; LORENZI; SOUZA, 2001).

Hemerocallis, ou lírios, como são popularmente conhecidos, são plantas extremamente rústicas e de fácil adaptação. No Brasil, foram amplamente utilizadas por Roberto Burle Marx, em seus inúmeros projetos paisagísticos de cunho público e privado. Desta forma, são plantas que podem ser encontradas em diversas cidades brasileiras, constituindo-se até mesmo em um aspecto cultural.

Atualmente, devido aos diversos conhecimentos produzidos pelo melhoramento genético, muitos híbridos de Hemerocallis foram desenvolvidos, mas ainda muito pouco se sabe a respeito de sua fisiologia e especialmente em relação ao manejo de seu florescimento, que ocorre somente na primaveraverão. Esse é um fator que pode ser preponderante aos produtores que desejam obter plantas floridas o ano inteiro ou ainda para que possam manejar o florescimento em sua produção, de forma a melhorar os atributos comercias de seus produtos e manter um fluxo constante de produção de mudas floridas. Desta forma os reguladores vegetais apresentam-se como uma ferramenta importante e menos onerosa para os produtores de flores.

De todos os reguladores vegetais que têm sido aplicados em plantas sob condições não indutivas, apenas as giberelinas (GAs) têm demonstrado causar efetiva formação de flores numa grande variedade de espécies (ZEEVART, 1971).

Dentro desse contexto, o objetivo do presente trabalho foi estudar a indução do florescimento em Hemerocalis hybrida cultivar Graziela Barroso, pela aplicação de diferentes concentrações de ácido giberélico $\left(\mathrm{GA}_{3}\right)$, nas estações verão (janeiroabril), outono (abril-julho) e inverno (julhooutubro), de forma a aprimorar seus atributos para a comercialização.

\section{Material e métodos}

$\mathrm{O}$ ácido giberélico utilizado $\left(\mathrm{GA}_{3}\right)$ foi o produto comercial ProGibb ${ }^{\circledR}$, nas concentrações: $0 \mathrm{mgL}^{-1}$ $\mathrm{GA}_{3}$ - controle, apenas água destilada; $15 \mathrm{mgL}^{-1} \mathrm{GA}_{3}$ e $30 \mathrm{mgL}^{-1} \mathrm{GA}_{3}$, aplicados nas folhas das plantas, por intermédio de um pulverizador costal manual, com bico de aplicação tipo leque, utilizando-se um volume aproximado de $10 \mathrm{~mL}$ da solução por vaso.

Emjaneiro(verão), abril(outono)ejulho(inverno) do ano de 2005, 300 mudas, obtidas por meio de "divisão de touceira" (propagação vegetativa), por estação, com aproximadamente um ano de idade, com cerca de $20 \mathrm{~cm}$ de comprimento e $5 \mathrm{~cm}$ de diâmetro, foram plantadas em vasos de polietileno, com volume de 2,8 litros, preenchidos com substrato (NPK 10:10:10; esterco de ave; terra e casca de arroz carbonizada) na proporção 1:8:80:100. Estas mudas 
foram mantidas em estufa, (área total - $200 \mathrm{~m}^{2}$; área útil do experimento $150 \mathrm{~m}^{2}$, naturalmente iluminada com telado de plástico, com temperaturas médias, máxima no verão $32^{\circ} \mathrm{C}$, outono $26^{\circ} \mathrm{C}$, inverno $23^{\circ} \mathrm{C}$ e mínimas no verão $27^{\circ} \mathrm{C}$, outono $22^{\circ} \mathrm{C}$, inverno $20^{\circ}$ C, umidade relativa do ar no verão $78 \%$, outono $79 \%$ e inverno $80 \%$, as quais foram obtidas por meio de termômetro com bulbo seco e úmido e termômetro com temperatura máxima e mínima, a irrigação foi realizada manualmente vaso a vaso, com um volume gasto por vaso de aproximadamente 300 ml) por 70 dias em cada estação do ano, em área pertencente à Empresa Agrícola da Ilha, localizada em Joinville, Santa Catarina, latitude Sul 26 $18^{\prime} 05$ “, longitude oeste. 48 50'38”. (FUNDAÇÃO PESQUISA E PLANEJAMENTO PARA O DESENVOLVIMENTO SUSTENTÁVEL DE JOINVILLE - IPPUJ, 2005).

Semanalmente foram retiradas 30 plantas para análise das variáveis: número e comprimento de hastes florais emitidas, número de botões florais, massa seca total da planta e área foliar por planta.

Foi utilizado um delineamento experimental inteiramente casualizado com um fatorial $3 \times 10$, testando-se 30 tratamentos com cinco repetições, oriundos da combinação das três concentrações de $\mathrm{GA}_{3}$ e dez números de aplicações de $\mathrm{GA}_{3}$.

As aplicações foram realizadas semanalmente e tiveram início uma semana após as instalações dos experimentos, totalizando 10 aplicações por estação (sendo a aplicação de número 0 a testemunha, ou seja, nenhuma planta recebeu aplicação de $\mathrm{GA}_{3}$, finalizando na aplicação de número 9 , ou seja, plantas que receberam 9 aplicações de $\mathrm{GA}_{3}$ ), realizadas utilizando-se um pulverizador costal manual (capacidade de 7,6 litros), com bico de aplicação tipo leque (vazão de $0,32 \mathrm{l} / \mathrm{min}$ ), no período da manhã, entre 8:00 e 9:00 horas, utilizando-se um volume aproximado de $10 \mathrm{ml}$ da solução por vaso.
Desta forma, o experimento foi conduzido por 10 semanas para cada estação do ano.

\section{Resultados e discussão}

No experimento da estação verão, a análise de variância para o número de hastes por planta mostrou que não há diferenças entre as concentrações utilizadas de $\mathrm{GA}_{3}$, existindo apenas diferença entre os números de aplicações de $\mathrm{GA}_{3}$. O teste de comparação de médias mostra que o melhor número de aplicação é três, quando se produziu 0,73 hastes florais/planta, em média (Tabela 1).

Isto ocorreu, provavelmente, em virtude do próprio desenvolvimento das plantas, e não da ação do fitorregulador $\mathrm{GA}_{3}$, pois nas primeiras aplicações o número de hastes foi reduzido; aumentando e diminuindo novamente em virtude da mudança de fotoperíodo, uma vez que o experimento foi instalado no final do mês de janeiro, e a partir da sexta aplicação, que ocorreu na segunda quinzena do mês de março, já estava iniciando-se a inversão dos dias longos para os dias curtos. Segundo Agrícola da Ilha (2003), a cultivar Graziela Barroso possui um período de floração de outubro a fevereiro, meses que correspondem ao fotoperíodo indutivo (dias longos).

$O$ efeito de uma substância reguladora de crescimento, além de depender dos fatores ambientais, depende também da sua concentração, do número de aplicações, da época de aplicação, do estádio de crescimento da planta e da natureza da espécie ou cultivar tratada (COELHO; OLIVEIRA; CALDAS, 1983; ALMEIDA; PEREIRA, 1996).

King, Pharis e Mander (1987) dizem que, dependendo da espécie, o local e o modo de ação das giberelinas podem diferir, e isso pode explicar as respostas contraditórias observadas entre e dentro da mesma espécie. 
Tabela 1. Efeito das aplicações de $\mathrm{GA}_{3}$ no número de hastes emitidas por planta de Hemerocallis hybrida cv. Graziela Barroso no verão (janeiro-abril/2005).

\begin{tabular}{cccccccccc}
\hline \multicolumn{7}{c}{ Número de aplicações de $\mathrm{GA}_{3} /$ Número de hastes florais por planta } \\
\hline $0(03 / 02)$ & $1(11 / 02)$ & $2(20 / 02)$ & $3(26 / 02)$ & $4(05 / 03)$ & $5(12 / 03)$ & $6(19 / 03)$ & $7(01 / 04)$ & $8(08 / 04)$ & $9(16 / 04)$ \\
\hline $0,03 \mathrm{C}$ & $0,10 \mathrm{C}$ & $0,23 \mathrm{BC}$ & $0,73 \mathrm{~A}$ & $0,57 \mathrm{AB}$ & $0,27 \mathrm{BC}$ & $0,07 \mathrm{C}$ & $0,07 \mathrm{C}$ & $0 \mathrm{C}$ & $0 \mathrm{C}$ \\
\hline $\mathrm{CV} \%$ & 8,62 & & & & & & & & \\
Teste $\mathrm{F}$ & $0,190^{* *}$ & & & & & & & & \\
\hline
\end{tabular}

Médias seguidas pela mesma letra maiúscula na horizontal, não diferem estatisticamente pelo teste de Tukey a $5 \%$ de probabilidade. n.s - não significativo ** - significativo a $1 \%$ de probabilidade

No experimento da estação outono não houve emissão de hastes florais em nenhum dos tratamentos, fato que corrobora para a constatação da complexidade acerca do florescimento nos vegetais, ou seja, não é apenas um fator que faz com que uma planta entre em processo de florescimento e sim diversos fatores que, muitas vezes, atuam em sinergismo.

O florescimento é o resultado final de processos fisiológicos, seqüências bioquímicas e ação gênica, com todo o sistema responsável pela influência do estímulo ambiental ao longo do tempo. Certamente as relações entre alguns dos componentes deste sistema são obscuras e difíceis de serem traçadas (MURFET, 1977).

A maioria das plantas usa sinais do meio para regular a transição da fase vegetativa para a reprodutiva, completando a reprodução sexuada sob condições extremas favoráveis. Alguns fatores que exibem mudanças sazonais regulares controlam o florescimento, sendo os mais importantes o fotoperíodo, a temperatura e a disponibilidade de água (BERNIER, 1988). Neste caso para $H$. hybrida Graziela Barroso se faz salutar a questão do fotoperíodo, daí a intenção de substituir a quantidade de luz necessária para seu florescimento pelo indutor giberelina, no entanto as concentrações utilizadas parecem não ter causado a reversão do estádio juvenil para o maduro na espécie.

No experimento estação inverno, para a variável número de hastes por planta obteve-se resultado semelhante a estação verão, sendo os melhores números de aplicações de três a oito (Tabela 2). Novamente nota-se a questão do próprio desenvolvimento da planta e fotoperíodo indutivo, já comentados anteriormente. A partir da quarta aplicação, a inversão dos dias curtos para dias longos começa a acontecer gradualmente, o que corrobora, mais uma vez, com a citação de que Hemerocallis hybrida cv Graziela Barroso floresce a partir de outubro. Entretanto, nas duas últimas aplicações, o número de hastes foi inferior às demais e isto deve ter ocorrido em virtude da espécie preferir mais o cultivo a pleno sol do que no interior de uma estufa. Assim, observou-se ao final dos três experimentos que as plantas não apresentavam o mesmo vigor e também estavam muito suscetíveis ao ataque de pragas, principalmente afídeos.

Tabela 2. Efeito das aplicações de $\mathrm{GA}_{3}$ no número de hastes emitidas por planta de Hemerocallis hybrida cv. Graziela Barroso no inverno (julho-outubro/2005).

\begin{tabular}{cccccccccc}
\hline \multicolumn{7}{c}{ Número de aplicações de $\mathrm{GA}_{3} /$ Número de hastes florais por planta } \\
\hline $0(05 / 08)$ & $1(12 / 08)$ & $2(19 / 08)$ & $3(26 / 08)$ & $4(02 / 09)$ & $5(09 / 09)$ & $6(17 / 09)$ & $7(23 / 09)$ & $8(30 / 09)$ & $9(06 / 10)$ \\
\hline $0,00 \mathrm{D}$ & $0,00 \mathrm{D}$ & $0,03 \mathrm{CD}$ & $0,27 \mathrm{ABC}$ & $0,53 \mathrm{~A}$ & $0,67 \mathrm{~A}$ & $0,53 \mathrm{~A}$ & $0,70 \mathrm{~A}$ & $0,50 \mathrm{AB}$ & $0,20 \mathrm{BCD}$ \\
\hline $\mathrm{CV} \%$ & 10,75 & & & & & & & & \\
Teste $\mathrm{F}$ & $0,196^{* *}$ & & & & & & &
\end{tabular}

Médias seguidas pela mesma letra maiúscula na horizontal, não diferem estatisticamente pelo teste de Tukey a 5\% de probabilidade. n.s - não significativo $\quad * *$ - significativo a $1 \%$ de probabilidade 
Em relação à variável comprimento de haste por planta $(25,80 \mathrm{~cm}$ - maior valor encontrado na terceira semana de aplicação em janeiro-abril; 17,73 $\mathrm{cm}$ - maior valor encontrado na sétima aplicação em julho-outubro) e número de botões florais $(2,93$ botões - maior valor encontrado na terceira semana de aplicação em janeiro-abril; 2,10 botões - maior valor encontrado na sétima aplicação em julhooutubro), tanto no experimento da estação verão quanto no experimento da estação inverno não houve interação entre os fatores estudados, e nem diferenças estatísticas significativas para o fator concentrações de $\mathrm{GA}_{3}$, mostrando mais uma vez que a utilização do ácido giberélico $\left(\mathrm{GA}_{3}\right)$ tornou-se dispensável para incremento dessas variáveis.

No experimento da estação verão, a variável massa seca apresentou interação entre os fatores estudados e, por meio do teste de comparação de médias, observou-se que para as três concentrações de $\mathrm{GA}_{3}$, o melhor número de aplicações foi nove, sendo de 10,54; 11,14 e 13,81 g, respectivamente para as três concentrações de $\mathrm{GA}_{3}$ (Tabela 3). Entretanto, este valor não justifica a utilização do ácido giberélico para melhorar ou aumentar o crescimento da espécie em questão uma vez que em números absolutos a diferença para esta variável entre a testemunha e as concentrações $15 \mathrm{mgL}^{-1} \mathrm{e}$ $30 \mathrm{mgL}^{-1}$ de $\mathrm{GA}_{3}$ é pequena.

$\mathrm{Na}$ espécie nativa medicinal Lippia alba (ervacidreira brasileira), a produtividade também não foi significativamente aumentada com a utilização de $\mathrm{GA}_{3}$ (STEFANINI; RODRIGUES; MING, 1998).

Tabela 3. Efeito das aplicações de $\mathrm{GA}_{3}$ na massa seca total (g) por planta de Hemerocallis hybrida cv. Graziela Barroso no verão (janeiro-abril/2005).

\begin{tabular}{|c|c|c|c|c|c|c|c|c|c|c|}
\hline \multirow{2}{*}{$\begin{array}{l}\text { Do s e s } \\
\text { de } \mathrm{GA}_{3}\end{array}$} & \multicolumn{10}{|c|}{ Aplicações de $\mathrm{GA}_{3} /$ Massa seca total (g) por planta } \\
\hline & $0(03 / 02)$ & $1(11 / 02)$ & $2(20 / 02)$ & $3(26 / 02)$ & $4(05 / 03)$ & $5(12 / 03)$ & $6(19 / 03)$ & $7(01 / 04)$ & $8(08 / 04)$ & $9(16 / 04)$ \\
\hline $0 \mathrm{mgL}^{-1}$ & 5,80Acde & 5,28Ade & $4,52 \mathrm{Ae}$ & 5,81Acde & $6,37 \mathrm{Abcd}$ & $4,77 \mathrm{Ae}$ & $6,56 \mathrm{Abcd}$ & $6,94 \mathrm{Abc}$ & $7,86 \mathrm{Ab}$ & $10,54 \mathrm{Aa}$ \\
\hline $15 \mathrm{mgL}^{-1}$ & 5,40Ade & 5,38Ade & $5,28 \mathrm{Ae}$ & $6,83 \mathrm{Abcd}$ & 5,51Ade & 6,12 Acde & $7,52 \mathrm{Abc}$ & $7,78 \mathrm{Ab}$ & 6,33Abcde & $11,14 \mathrm{Aa}$ \\
\hline $30 \mathrm{mgL}^{-1}$ & 5,57Ad & $5,32 \mathrm{Ad}$ & $5,75 \mathrm{Acd}$ & $4,75 \mathrm{Ad}$ & $5,29 \mathrm{Ad}$ & 5,99Acd & $6,13 \mathrm{Acd}$ & $7,22 \mathrm{Abc}$ & $8,61 \mathrm{Ab}$ & $13,81 \mathrm{Aa}$ \\
\hline CV\% & 11,04 & & & & & & & & & \\
\hline Teste F & $3,902^{* *}$ & & & & & & & & & \\
\hline
\end{tabular}

Médias seguidas pela mesma letra maiúscula na vertical (fator concentração de $\mathrm{GA}_{3}$ ) e minúscula na horizontal (fator número de aplicações de $\mathrm{GA}_{3}$ ), não diferem estatisticamente pelo teste de Tukey a $5 \%$ de probabilidade.

ns - não significativo

** - significativo a $1 \%$ de probabilidade

No experimento da estação outono, a variável massa seca por planta não apresentou interação entre os fatores estudados; somente houve significância para o fator número de aplicações de $\mathrm{GA}_{3}$, o qual, por meio do teste de comparação de médias, mostrou que não houve diferença estatística entre as médias, embora o valor absoluto da última aplicação seja o maior, de 8,58 g (Tabela 4). Esta informação também pode ser considerada previsível, uma vez que à medida que se passa o tempo, a planta vai crescendo e aumentando sua biomassa, muito embora nessa estação haja um repouso no crescimento dos vegetais. 
Tabela 4. Efeito das aplicações de $\mathrm{GA}_{3}$ na massa seca total (g) por planta de Hemerocallis hybrida cv. Graziela Barroso no outono (abril-julho/2005).

\begin{tabular}{cccccccccc}
\hline \multicolumn{7}{c}{ Aplicações de $\mathrm{GA}_{3} /$ Massa seca total (g) por planta } \\
\hline $0(29 / 04)$ & $1(07 / 05)$ & $2(14 / 05)$ & $3(21 / 05)$ & $4(27 / 05)$ & $5(03 / 06)$ & $6(10 / 06)$ & $7(17 / 06)$ & $8(24 / 06)$ & $9(01 / 07)$ \\
\hline $4,96 \mathrm{~B}$ & $4,62 \mathrm{~B}$ & $4,19 \mathrm{~B}$ & $4,52 \mathrm{~B}$ & $3,80 \mathrm{~B}$ & $4,16 \mathrm{~B}$ & $5,00 \mathrm{~B}$ & $4,60 \mathrm{~B}$ & $5,57 \mathrm{~B}$ & $8,58 \mathrm{~A}$ \\
\hline $\mathrm{CV} \%$ & 39,64 & & & & & & & & \\
Teste F & $27,535^{* *}$ & & & & & & & \\
\hline
\end{tabular}

Médias seguidas pela mesma letra maiúscula na horizontal, não diferem estatisticamente pelo teste de Tukey a $5 \%$ de probabilidade.

n.s - não significativo

** - significativo a $1 \%$ de probabilidade

E no experimento da estação inverno, a mesma variável também não mostrou interações significativas entre os fatores estudados, apenas apresentou diferenças para o fator número de aplicações de $\mathrm{GA}_{3}$, fato explicado pelo próprio desenvolvimento da planta.

Existem trabalhos que obtiveram resultados discordantes, como é o caso da utilização do $\mathrm{GA}_{3}$ juntamente com Daminozide, uma substância inibidora da biossíntese de giberelina endógena, combinados na quantidade de 50 ppm e 3500 ppm, respectivamente, em Chrysantemum morifolium cv "Bright Golden Anne". A massa seca neste estudo para este tratamento foi superior à do controle, 7,5 g e 6,0 g, respectivamente (KAMBALAPALLY; MAKI; RAJAPAKSE, 2005).

Para a variável área foliar, no experimento da estação verão não houve interação entre os fatores estudados, apenas diferenças significativas para o fator número de aplicações de $\mathrm{GA}_{3}$, sendo o melhor resultado, em valor absoluto, encontrado na nona aplicação de $394,90 \mathrm{~cm}^{2}$ (Tabela 5).

Tabela 5. Efeito das aplicações de $\mathrm{GA}_{3}$ na área foliar $\left(\mathrm{cm}^{2}\right)$ por planta de Hemerocallis hybrida cv. Graziela Barroso em janeiro-abril/2005.

\begin{tabular}{|c|c|c|c|c|c|c|c|c|c|}
\hline \multicolumn{10}{|c|}{ Aplicações de $\mathrm{GA}_{3} /$ Área foliar por planta $\left(\mathrm{cm}^{2}\right)$} \\
\hline $0(03 / 02)$ & $1(11 / 02)$ & $2(20 / 02)$ & $3(26 / 02)$ & $4(05 / 03)$ & $5(12 / 03)$ & $6(19 / 03)$ & $7(01 / 04)$ & $8(08 / 04)$ & $9(16 / 04)$ \\
\hline $121,20 \mathrm{DE}$ & $102,72 \mathrm{D}$ & $94,15 \mathrm{D}$ & $128,80 \mathrm{D}$ & $201,80 \mathrm{C}$ & $222,00 \mathrm{C}$ & $291,40 \mathrm{~B}$ & $332,20 \mathrm{AB}$ & $355,60 \mathrm{~A}$ & $394,90 \mathrm{~A}$ \\
\hline $\mathrm{CV} \%$ & 1,58 & & & & & & & & \\
\hline Teste F & 520926,74 & $5 * *$ & & & & & & & \\
\hline
\end{tabular}

Médias seguidas pela mesma letra minúscula na horizontal, não diferem estatisticamente pelo teste de Tukey a $5 \%$ de probabilidade.

n.s - não significativo

** - significativo a $1 \%$ de probabilidade

Na estação outono, a variável área foliar por planta apresentou interação entre os fatores estudados, sendo os melhores resultados encontrados, para a concentração $0 \mathrm{mgL}^{-1}$ de $\mathrm{GA}_{3}, 408,4 \mathrm{~cm}^{2}$ na sexta aplicação; para $15 \mathrm{mgL}^{-1}$ de $\mathrm{GA}_{3}, 405,5 \mathrm{~cm}^{2}$ na sexta aplicação e para $30 \mathrm{mgL}^{-1} \mathrm{deGA}_{3}, 436,30 \mathrm{~cm}^{2}$ na nona aplicação (Tabela 6); na estação inverno não houve interação significativa para os fatores estudados, apenas diferenças significativas para o fator número de aplicações de $\mathrm{GA}_{3}$. Na estação inverno, o melhor resultado foi encontrado no último número de aplicações, $355,6 \mathrm{~cm}^{2}$. 
Isto implica na constatação de que embora na estação outono as plantas pulverizadas com o ácido giberélico possam ter mostrado melhor desempenho no quesito área foliar, não determina a sua utilização, nas concentrações testadas, para aprimorar o crescimento de Hemerocallis hybrida cv Graziela Barroso, visto que o maior atrativo comercial da espécie encontra-se em sua haste floral, em termos de custo-benefício utilizar o fitoregulador apenas para aumento de seu crescimento não seria interessante aos produtores.

No mesmo estudo citado anteriormente utilizando Chrysantemum morifolium cv "Bright Golden Anne", os autores também obtiveram aumento para a variável área foliar no tratamento que combinava Daminozide e $\mathrm{GA}_{3}, 1033,1 \mathrm{~cm}^{2}$, nas plantas submetidas a três pulverizações dessa combinação, na primavera e verão, contra $939,4 \mathrm{~cm}^{2}$ de área foliar para plantas do controle (KAMBALAPALLY; MAKI; RAJAPAKSE, 2005).
Fatores ambientais, principalmente luz e temperatura devem estar intimamente envolvidos na sensibilidade de Hemerocallis hybrida cv. Graziela Barroso, já que foram obtidos resultados diferentes nas três estações. E, ainda, as grandes mudanças de clima no ano (maior ou menor luminosidade e maior ou menor temperatura) afetam a produção, mesmo dentro de estufas (AKI; PEROSA, 2002).

Recomendam-se desta forma, novos estudos acerca do seu florescimento, a fim de se entender como e porque a espécie floresce apenas em determinada época do ano (primavera/verão), uma vez que o florescimento é o resultado da interação de diversos fatores, ou ainda recomenda-se a pesquisa de outros atributos comerciais que poderiam ser aprimorados. Além disto, seria interessante a troca de informações e pesquisas com outros atores da cadeia produtiva de flores e plantas ornamentais, com varejistas, atacadistas e até mesmo, consumidores finais.

Tabela 6. Efeito das aplicações de $\mathrm{GA}_{3}$ na área foliar $\left(\mathrm{cm}^{2}\right)$ por planta de Hemerocallis hybrida $\mathrm{cv}$. Graziela Barroso em abril-julho/2005.

\begin{tabular}{|c|c|c|c|c|c|c|c|c|c|c|}
\hline \multirow{2}{*}{$\begin{array}{l}\text { Doses } \\
\text { de GA }\end{array}$} & \multicolumn{10}{|c|}{ Aplicações de $\mathrm{GA}_{3} /$ Área foliar por planta $\left(\mathrm{cm}^{2}\right)$} \\
\hline & $0(29 / 04)$ & $1(07 / 05)$ & $2(14 / 05)$ & $3(21 / 05)$ & $4(27 / 05)$ & $5(03 / 06)$ & $6(10 / 06)$ & $7(17 / 06)$ & $8(24 / 06)$ & $9(01 / 07)$ \\
\hline $0 \mathrm{mg} \mathrm{L}^{-1}$ & $210,4 \mathrm{Aa}$ & $264,9 \mathrm{Aab}$ & $301,4 \mathrm{Aab}$ & $306,5 \mathrm{Aab}$ & $347,5 \mathrm{Aab}$ & 396,8Aa & $408,4 \mathrm{Aa}$ & $386,9 \mathrm{Aa}$ & $344,4 \mathrm{Aab}$ & $397,2 \mathrm{Aa}$ \\
\hline $15 \mathrm{mg} \mathrm{L}^{-1}$ & $111,9 \mathrm{Cd}$ & $169,4 \mathrm{Ccd}$ & $144,1 \mathrm{Ccd}$ & $\begin{array}{l}182,3 \\
\text { Cbcd }\end{array}$ & $300,7 \mathrm{Cabc}$ & $348,2 \mathrm{Cab}$ & $405,5 \mathrm{Ca}$ & $287,5 \mathrm{Cabc}$ & $299,9 \mathrm{Cab}$ & $393,1 \mathrm{Ca}$ \\
\hline $30 \mathrm{mg} \mathrm{L}^{-1}$ & $145,7 \mathrm{Bb}$ & $198,0 \mathrm{Bb}$ & $189,9 \mathrm{Bb}$ & $175,6 \mathrm{Bb}$ & $292,7 \mathrm{Bab}$ & $398,9 \mathrm{Ba}$ & $434,5 \mathrm{Ba}$ & $399,1 \mathrm{Ba}$ & $380,3 \mathrm{Ba}$ & $436,3 \mathrm{Ba}$ \\
\hline $\mathrm{CV} \%$ & 1,23 & & & & & & & & & \\
\hline Teste F & 6490,295 & & & & & & & & & \\
\hline
\end{tabular}

Médias seguidas pela mesma letra maiúscula na vertical (fator concentração de $\mathrm{GA}_{3}$ ) e minúscula na horizontal (fator número de aplicações de $\mathrm{GA}_{3}$ ), não diferem estatisticamente pelo teste de Tukey a $5 \%$ de probabilidade.

n.s - não significativo

** - significativo a $1 \%$ de probabilidade

\section{Conclusões}

Não se pode indicar a utilização do ácido giberélico nas concentrações estudadas como uma ferramenta para aprimoramento de atributos comerciais (florescimento e crescimento) em Hemerocallis hybrida cv Graziela Barroso; além disto, a espécie não apresenta desenvolvimento adequado em estufa. 


\section{Referências}

AGRÍCOLA DA ILHA. Hemerocallis: catálogo 2003. Joinville: Agrícola da Ilha, 2003.

AKI, A.; PEROSA, J. M. Y. Aspectos da produção e consumo de flores e plantas ornamentais no Brasil. Revista Brasileira de Horticultura Ornamental, Campinas, v. 8, n. 1/2, p. 13-23, 2002.

ALMEIDA, J. A. S.; PEREIRA, M. F. D. A. The control of flower initiation by gibberellin in Helianthus annuus. Plant Growth Regulation, Dordrecht, v. 19, n. 2, p. 109115, 1996.

BERNIER, G. The control of floral evocation and morphogenesis. Annual Review of Plant Physiology and Plant Molecular Biology, Palo Alto, v. 39, p. 175-219, 1988.

CASTAN, J. O setor de paisagismo em Santa Catarina. In: AKI, A. (Ed.). Bússola da comercialização para produtores de ornamentais. Bandeirantes: Heliza, 2002. p. 129-132.

COELHO, Y. S.; OLIVEIRA, A. A. R.; CALDAS, R. C. Efeitos do ácido giberélico (AG) no crescimento de portaenxertos para citrus. Pesquisa Agropecuária Brasileira, Brasília, v. 18, n. 11, p. 1229-1232, 1983.

FUNDAÇÃO PESQUISA E PLANEJAMENTO PARA O DESENVOLVIMENTO SUSTENTÁVEL DE JOINVILLE - IPPUJ. Joinville - Cidade em Dados. Disponível em: <http://www.ippuj.sc.gov.br/index.php ?goto $=$ conteudo $\&$ menu $=3 \&$ submenu $=33>$. Acesso em: 12 set. 2005.
HERTOGH, A. A.; LE NARD, M. The physiology of flower bulbs. Amsterdam: Elsevier Science Publishers B. V., 1993.

KAMBALAPALLY, V. R.; MAKI, S. L.; RAJAPAKSE, N. C. Influence of gibberellic acid and copper sulfate filters on growth of Chrysanthemum. Disponivel em: $<$ www.clemson.edu/hort/sctop/bsec/bsec.07.pdf $>$. Acesso em: 22 nov. 2005.

KING, R. W.; PHARIS, R. P.; MANDER, L. N. Gibberellins in relation to growth and flowering in Pharbitis nil Chois. Plant Physiology, Rockville, v. 84, n. 4, p. 1126-1131, 1987.

LORENZI, H.; SOUZA, H. M. Plantas ornamentais no Brasil. Arbustivas, herbáceas e trepadeiras. 3. ed. Nova Odessa: Instituto Plantarum, 2001.

MURFET, I. C. Environmental interaction and the genetics of flowering. Annual Review of Plant Physiology, Palo Alto, v. 28, p. 253-278, June 1977.

STEFANINI, M. B.; RODRIGUES, S. D.; MING, L. C. Efeito do ácido giberélico, ethephon e CCC nos índices da análise de crescimento (A.F.E., R.A.F. e R.M.F.) em erva-cidreira brasileira. Revista Brasileira de Plantas Medicinais, Botucatu, v. 1, n.1, p. 15-22, 1998.

ZEEVART, J. A. D. Effects of photoperiod on growth rate and endogenous gibberellins in the long-day rosette plant spinach. Plant Physiology, Minneapolis, v. 47, n. 6, p. 821-827, 1971. 